\title{
Effect of bariatric surgery on sulphur amino acids and glutamate
}

\author{
Erlend T. Aasheim ${ }^{1,2,3,4_{*}}$, Amany K. Elshorbagy ${ }^{5}$, Lien My Diep ${ }^{6}$, Torgeir T. Søvik ${ }^{7}$, Tom Mala ${ }^{7}$, \\ Maria Valdivia-Garcia ${ }^{5}$, Torsten Olbers ${ }^{8}$, Thomas Bøhmer ${ }^{1}$, Kåre I. Birkeland ${ }^{1,3}$ and Helga Refsum ${ }^{5,9}$ \\ ${ }^{1}$ Department of Endocrinology, Oslo University Hospital Aker, Trondheimsv 235, 0514 Oslo, Norway \\ ${ }^{2}$ Hormone Laboratory, Oslo University Hospital Aker, Trondheimsv 235, 0514 Oslo, Norway \\ ${ }^{3}$ Faculty of Medicine, University of Oslo, Sognsvannsv 9, 0315 Oslo, Norway \\ ${ }^{4}$ Imperial Weight Centre, Imperial College London, London, UK \\ ${ }^{5}$ Department of Pharmacology, Oxford University, Mansfield Road, Oxford OX1 3QT, UK \\ ${ }^{6}$ Research Centre, Oslo University Hospital Aker, Trondheimsv 235, 0514 Oslo, Norway \\ ${ }^{7}$ Department of Gastrointestinal Surgery, Oslo University Hospital Aker, Trondheimsv 235, 0514 Oslo, Norway \\ ${ }^{8}$ Department of Surgery and Gastrosurgical Research, Sablgrenska University Hospital, Box 100, 40530 Göteborg, Sweden \\ ${ }^{9}$ Department of Nutrition, Institute of Basic Medical Sciences, University of Oslo, Sognsvannsv 9, 0316 Oslo, Norway \\ (Received 16 August 2010-Revised 1 December 2010-Accepted 21 December 2010-First published online 4 May 2011)
}

\begin{abstract}
Plasma total cysteine (tCys) concentrations are associated with BMI. To study the relationship between tCys and BMI, we monitored the changes in serum concentrations of tCys and metabolically related compounds in sixty obese patients (BMI $50-60 \mathrm{~kg} / \mathrm{m}^{2}$ ) from before to 1 year after either gastric bypass surgery (mean $30 \%$ weight loss) or duodenal switch surgery (mean $41 \%$ weight loss). A total of fifty-eight healthy persons (BMI $17-31 \mathrm{~kg} / \mathrm{m}^{2}$ ) served as controls. Before surgery, obese patients had modestly (approximately $17 \%$ ) higher mean serum tCys, and markedly ( $>2$-fold) higher glutamate concentrations, than controls ( $P \leq 0 \cdot 001$ for both). Serial examinations after surgery revealed that gastric bypass patients had no change in tCys concentrations $(P=0 \cdot 22)$, while duodenal switch patients showed a modest (approximately $12 \%$ ) but significant decrease in tCys $(P<0 \cdot 001)$. Total homocysteine concentrations increased in duodenal switch patients but not in gastric bypass patients. Independent of surgery type, serum concentrations of methionine and cystathionine decreased $(P<0 \cdot 05$ for both), while serum glutathione and taurine remained stable. Glutamate concentrations declined, as did $\gamma$-glutamyltransferase activity $(P<0.001$ for both). These results show that despite 30\% weight loss, and decreases in methionine, cystathionine and glutamate, there was no significant change in serum tCys in patients after gastric bypass surgery. The decrease in tCys in patients undergoing duodenal switch could be related to malabsorption. The present findings do not suggest that BMI is a causal determinant of plasma tCys.
\end{abstract}

Key words: Obesity surgery: Homocysteine: Folic acid: Cobalamin: Pyridoxine

The sulphur amino acids (SAA) are fundamental to protein synthesis. Both methionine and cysteine are proteinogenic amino acids; methionine is required for translation of all eukaryotic proteins, and cysteine contributes to protein structure by forming disulphide bonds ${ }^{(1)}$. Although it has not been established that SAA can contribute to the development of obesity, methionine and cysteine intakes can promote growth and weight gain in mammals ${ }^{(2,3)}$. In humans, plasma total cysteine (tCys) levels are positively associated with BMI and obesity ${ }^{(4-6)}$, mediated through fat mass ${ }^{(6)}$. Among men and women in a population-based study, an increase from low to high tCys quintile was associated with an increase in fat mass of 6-9 kg, after adjustment for age, diet, lifestyle factors and serum lipids. Longitudinal studies have revealed that an increase or decrease in tCys over 6 years was linked with corresponding changes in BMI and fat mass ${ }^{(6,7)}$.

Obesity is also associated with changes in compounds metabolically related to cysteine. Plasma glutathione (GSH) correlates negatively with $\mathrm{BMI}^{(8)}$, while the amino acid glutamate is elevated in the blood $^{(9)}$ and plasma ${ }^{(10)}$ of obese individuals. Glutamate is linked to cysteine via GSH metabolism: cysteine and glutamate combine to form $\gamma$-glutamylcysteine in the rate-limiting step of GSH synthesis ${ }^{(11)}$. The membrane enzyme $\boldsymbol{\gamma}$-glutamyltransferase (GGT) hydrolyses GSH, with the subsequent release of cysteine and glutamate ${ }^{(12)}$. Serum GGT activity is elevated in obese persons ${ }^{(5,13)}$, and decreases

Abbreviations: BCAA, branched-chain amino acids; GGT, $\boldsymbol{\gamma}$-glutamyltransferase; GSH, glutathione; SAA, sulphur amino acids; tCys, total cysteine; tGSH, total glutathione; tHcy, total homocysteine.

*Corresponding author: Dr E. T. Aasheim, fax +44 2033130673, email e.t.aasheim@medisin.uio.no 
with weight loss ${ }^{(14)}$. Conversely, plasma concentrations of the cysteine precursor, homocysteine, often increase after weight loss ${ }^{(15)}$.

Despite the diverse evidence pointing to a link between cysteine metabolism and body weight, the effect of weight loss on cysteine metabolism is largely unexplored. The causal direction for the relationship between tCys and fat mass remains uncertain: does tCys promote body fat accumulation; or does fat mass influence cysteine production, catabolism, uptake or release? In favour of tCys promoting fat accumulation, intake of cysteine-rich diets has been associated with weight gain both in animal models ${ }^{(16,17)}$ and in patients with cancer ${ }^{(18)}$. However, recent studies on the role of adipose tissue in regulating cysteine conversion to taurine ${ }^{(19)}$ have suggested that adipose tissue mass could be an important determinant of cysteine and taurine levels. The hypothesis that fat mass determines plasma tCys has not been addressed in humans. Severely obese patients undergoing bariatric surgery may be an ideal population for testing the hypothesis: if fat mass determines tCys, then tCys concentrations would be expected to be markedly elevated before and drop significantly after bariatric surgery due to major weight loss. We, therefore, studied the effects of bariatric surgery in obese patients on serum concentrations of tCys and metabolically related compounds, such as methionine, homocysteine, cystathionine, taurine, GSH and glutamate, and serum GGT activity.

\section{Experimental methods}

The patients have been described in detail previously ${ }^{(20)}$. Briefly, sixty obese patients aged 20-50 years, from two centres (Oslo University Hospital Aker, $n$ 30; Sahlgrenska University Hospital, $n$ 30), were included in a randomised trial of gastric bypass and duodenal switch for weight loss (February 2006-August 2007; ClinicalTrials.gov identifier: NCT00327912). All patients had a BMI between 50 and $60 \mathrm{~kg} / \mathrm{m}^{2}$. In addition to the baseline visit, the patients were examined 6 weeks, 6 months and 1 year after surgery. Patients followed a $4184 \mathrm{~kJ}$ ( $1000 \mathrm{kcal})$ diet for 3 weeks before surgery. During surgery, the alimentary limb, biliopancreatic limb and common channel were created as follows: gastric bypass $150,50 \mathrm{~cm}$ and variable; duodenal switch $-200 \mathrm{~cm}$, variable and $100 \mathrm{~cm}^{(20)}$. After surgery, all patients were daily prescribed $\mathrm{Fe}, \mathrm{Ca}$, vitamin $\mathrm{D}$ and multivitamin/mineral supplements (selected contents: $2 \mathrm{mg}$ vitamin $\mathrm{B}_{6}, 200 \mu \mathrm{g}$ folic acid and $1 \mathrm{mg}$ vitamin $\mathrm{B}_{12}$ (Nycoplus multi; Nycomed, Asker, Norway)). Gastric bypass patients also received cyanocobalamin supplementation (intramuscular injections, $1 \mathrm{mg}$ every 3 months (Betolvex; Actavis, Oslo, Norway) or oral supplement, $1 \mathrm{mg}$ daily (Behepan; Pfizer, Sollentuna, Sweden)). Ursodeoxycholic acid was given until 6 months after surgery. Duodenal switch patients were more often taking additional fat-soluble vitamin supplements, but otherwise, supplement use was similar in the two groups ${ }^{(20)}$.

The controls were fifty-eight healthy men and women with BMI between 17 and $31 \mathrm{~kg} / \mathrm{m}^{2}$ and aged $19-59$ years, recruited January-May 2007 at Oslo University Hospital
Aker ${ }^{(21)}$. The controls were recruited as a convenience sample, with no attempt made to match controls and patients. Exclusion criteria were chronic disease and regular medication or multivitamin use. Persons using contraceptive medication ( $n$ 10) or thyroxine substitution $(n 4)$ were included. As a population-based control group for plasma tCys and BMI comparisons (see Fig. S1 of the supplementary material, available online at http://www.journals.cambridge.org/bjn), we used the Hordaland Homocysteine Study participants ( $n$ 3721, men and women aged 47-49 years) ${ }^{(6)}$.

The study was conducted according to the guidelines laid down in the Declaration of Helsinki, and all procedures involving human subjects were approved by the appropriate regional ethics committees for medical research. Written informed consent was obtained from all subjects.

\section{Biochemical analysis}

Overnight fasting blood samples were clotted for $30 \mathrm{~min}$ at room temperature, and serum was separated by centrifugation. Samples collected at Oslo University Hospital Aker were frozen and stored at $-80^{\circ} \mathrm{C}$ until assayed. Samples collected at Sahlgrenska University Hospital were thawed once for the analysis of 25-hydroxyvitamin $\mathrm{D}^{(20)}$ and were subsequently stored at $-20^{\circ} \mathrm{C}$ until assayed. Assays were performed within 2 years of blood sampling.

Serum concentrations of SAA and glutamate were analysed by liquid chromatography-tandem MS using modifications of described methods ${ }^{(16,22)}$. Methionine, total homocysteine (tHcy), cystathionine, tCys and total glutathione (tGSH) were analysed in a single run. A separate assay was used to simultaneously measure taurine and glutamate. Inter-assay CV was $<4 \%$ for tCys, tHcy and taurine; $<8 \%$ for methionine, cystathionine and $\mathrm{tGSH}$; and $10.5 \%$ for glutamate. Serum cobalamin was determined using Lactobacillus leichmannii microbiological assays ${ }^{(23)}$.

Serum creatinine, alanine aminotransferase, GGT and folate were measured during the follow-up with Hitachi (717 or 800) Modular multianalysers (Boehringer Mannheim, Mannheim, Germany $)^{(20)}$. Serum pyridoxal-5'-phosphate (vitamin $\mathrm{B}_{6}$ ) was measured by HPLC (Chromsystems, Munich, Germany). Plasma tHcy concentrations measured during the follow-up in some patients (using Hitachi 717 multianalyser) correlated well with the reported serum concentrations obtained by liquid chromatography-tandem MS (Pearson's $r \quad 0.95$, $P<0.001 ; n$ 18).

In total, frozen serum samples were available from 223 out of 240 study visits (93\%). As outlined above, serum handling and storage varied between the study sites (Oslo and Sahlgrenska). In agreement with our laboratory's experience that sample processing and freeze-thaw cycles may influence assay values, the serum concentrations for methionine, taurine, tGSH and glutamate differed markedly between the centres. We, therefore, report data on those metabolites only for patients with specimens kept at $-80^{\circ} \mathrm{C}$ ( $n$ 30; followed in Oslo), and data on tHcy, cystathionine and tCys from all patients $(n 60)$. 


\section{Statistical analysis}

For cross-sectional analyses, we compared patients and controls separately for men and women, using Fisher's exact test for proportions and unpaired Student's $t$ tests for continuous data. These tests, correlation analyses and multiple linear regressions were calculated using SPSS 14.0 (SPSS, Inc., Chicago, IL, USA).

For longitudinal analyses, we used linear mixed-effects models (with a random intercept and assuming compound symmetric correlation structure) for continuous dependent variables to assess a trend (time effect) across the four time points in each patient. Estimates of fixed-effects coefficients and their standard errors were extracted after fitting the model. Standardised values of the outcome variables were computed as predicted values from the model. These predictions were done for each time point in a new dataset, where potential confounders (age, sex and type of surgery) were replaced by their overall average values in the full dataset. Standard errors of the predicted values were estimated by assuming the predictors as nonrandom and using the estimated variance-covariance matrix for the fixed-effects estimates. Non-normally distributed variables (including tHcy, cystathionine, cobalamin, creatinine, alanine aminotransferase and GGT) were log-transformed before analysis. Data are expressed as standardised means or geometric means with 95\% CI. Linear mixed-effects models (lme function in the R-library nmle) were computed in R 2.8.1 for Windows ${ }^{(24)}$. The $P$ values are two-sided, and the significance level was 0.05 .

\section{Results}

\section{Participant characteristics}

Most participants (97\%) were of North European descent. Mean age was not significantly different between patients and controls, who had a mean BMI of 55 and $24 \mathrm{~kg} / \mathrm{m}^{2}$. Patients had lower serum pyridoxal- 5 -phosphate and folate concentrations than controls (Table 1).

\section{Total cysteine and related compounds in obese patients and healthy controls}

Before surgery, the severely obese patients had significantly but modestly (approximately 17\%) higher serum tCys concentrations than controls. Compared with controls, the obese patients also had significantly higher serum concentrations of amino acids both upstream (tHcy) and downstream (taurine) of cysteine, as well as of glutamate (Table 1).

\section{Effect of bariatric surgery on total cysteine and related compounds}

In the same obese patients, serum tCys and related compounds were also measured 6 weeks, 6 months and 1 year after a bariatric surgical procedure, which was either gastric bypass or duodenal switch. Unless stated otherwise, patients in the two surgical groups had similar trends in biomarker

Table 1. Baseline characteristics of obese patients and healthy controls (Mean values and standard deviations)

\begin{tabular}{|c|c|c|c|c|c|c|c|c|c|c|}
\hline & \multicolumn{5}{|c|}{ Women } & \multicolumn{5}{|c|}{ Men } \\
\hline & \multicolumn{2}{|c|}{ Controls ( $n 30)$} & \multicolumn{2}{|c|}{ Patients ( $n$ 42) } & \multirow[b]{2}{*}{$P^{*}$} & \multicolumn{2}{|c|}{ Controls (n 28) } & \multicolumn{2}{|c|}{ Patients ( $n$ 18) } & \multirow[b]{2}{*}{$P^{*}$} \\
\hline & Mean & SD & Mean & SD & & Mean & SD & Mean & SD & \\
\hline Age (years) & 39 & 11 & $35 \dagger$ & 7 & 0.08 & 39 & 11 & 37 & 5 & 0.43 \\
\hline BMI $\left(\mathrm{kg} / \mathrm{m}^{2}\right)$ & 23 & 3 & 55 & 4 & $<0.001$ & 25 & 3 & 55 & 3 & $<0.001$ \\
\hline Current smoker & & & & & 0.002 & & & & & 0.28 \\
\hline$n$ & \multicolumn{2}{|c|}{1} & \multicolumn{2}{|c|}{14} & & \multicolumn{2}{|c|}{4} & \multicolumn{2}{|c|}{5} & \\
\hline$\%$ & \multirow{2}{*}{\multicolumn{2}{|c|}{3}} & \multirow{2}{*}{\multicolumn{2}{|c|}{33}} & & \multirow{2}{*}{\multicolumn{2}{|c|}{14}} & \multirow{2}{*}{\multicolumn{2}{|c|}{28}} & \\
\hline Type 2 diabetes & & & & & 0.04 & & & & & 0.02 \\
\hline$n$ & \multicolumn{2}{|c|}{0} & \multicolumn{2}{|c|}{7} & & \multirow{2}{*}{\multicolumn{2}{|c|}{$\begin{array}{l}0 \\
0\end{array}$}} & \multicolumn{2}{|c|}{4} & \\
\hline$\%$ & \multicolumn{2}{|c|}{0} & \multicolumn{2}{|c|}{17} & & & & \multicolumn{2}{|c|}{22} & \\
\hline Creatinine $(\mu \mathrm{mol} / \mathrm{l})$ & 65 & 7 & 61 & 9 & 0.06 & 76 & 10 & 73 & 11 & 0.42 \\
\hline $\mathrm{ALT} †(\mathrm{U} / \mathrm{l})$ & 18 & 8 & 23 & 7 & 0.007 & 23 & 9 & 44 & 17 & $<0.001$ \\
\hline GGT† (U/l) & 15 & 7 & 43 & 33 & $<0.001$ & $23 \ddagger$ & 13 & 56 & 29 & $<0.001$ \\
\hline FAD (nmol/l) & 279 & 39 & 305 & 47 & 0.01 & 319 & 40 & 320 & 53 & 0.93 \\
\hline FMN (nmol/l) & 20 & 7 & 22 & 10 & 0.30 & 25 & 14 & 29 & 15 & 0.39 \\
\hline PLP (nmol/l) & 46 & 24 & 24 & 14 & $<0.001$ & 58 & 31 & 32 & 20 & 0.001 \\
\hline Folate† (nmol/l) & 16 & 4 & 13 & 6 & 0.01 & 16 & 5 & 12 & 4 & 0.04 \\
\hline Vitamin $\mathrm{B}_{12}(\mathrm{pmol} / \mathrm{l})$ & 305 & 104 & 419 & 694 & 0.65 & 364 & 103 & 344 & 139 & 0.37 \\
\hline tHcy $(\mu \mathrm{mol} / \mathrm{l})$ & $9 \cdot 3$ & $2 \cdot 1$ & 11.4 & $4 \cdot 8$ & 0.02 & $10 \cdot 3$ & $2 \cdot 1$ & 14.4 & $5 \cdot 8$ & 0.001 \\
\hline Cystathionine $(\mu \mathrm{mol} / \mathrm{l})$ & $0 \cdot 19$ & 0.09 & 0.22 & $0 \cdot 10$ & 0.13 & 0.22 & $0 \cdot 10$ & 0.30 & 0.17 & 0.03 \\
\hline tCys $(\mu \mathrm{mol} / \mathrm{l})$ & 257 & 28 & 303 & 40 & $<0.001$ & 275 & 34 & 320 & 50 & 0.001 \\
\hline Methionine† $(\mu \mathrm{mol} / \mathrm{l})$ & $22 \cdot 6$ & $2 \cdot 8$ & $20 \cdot 7$ & 1.9 & 0.007 & 24.4 & 4.0 & $24 \cdot 7$ & $2 \cdot 4$ & 0.84 \\
\hline $\mathrm{tGSH} \dagger(\mu \mathrm{mol} / \mathrm{l})$ & 4.7 & 0.8 & $5 \cdot 0$ & $1 \cdot 2$ & 0.27 & 5.5 & $1 \cdot 3$ & $5 \cdot 2$ & $1 \cdot 8$ & 0.57 \\
\hline Taurine† $(\mu \mathrm{mol} / \mathrm{l})$ & 59 & 14 & 81 & 17 & $<0.001$ & 47 & 13 & 79 & 21 & $<0.001$ \\
\hline Glutamate† $(\mu \mathrm{mol} / \mathrm{l})$ & 79 & 14 & 201 & 68 & $<0.001$ & $98 \ddagger$ & 25 & 227 & 58 & $<0.001$ \\
\hline
\end{tabular}

ALT, alanine aminotransferase; GGT, $\gamma$-glutamyltransferase; PLP, pyridoxal-5'-phosphate; tHcy, total homocysteine; tCys, total cysteine; tGSH, total glutathione.

* Comparisons by the $t$ test or Fisher's exact test. ALT, GGT, PLP, folic acid, vitamin $\mathrm{B}_{12}$, homocysteine and cystathionine were compared using log-transformed values.

$\dagger$ Data available only from the Norwegian patients in the study (twenty-two women and eight men).

$\ddagger$ One male control (outlier) was excluded from comparisons of GGT and glutamate. 
values during the follow-up (i.e. no significant time $\times$ procedure interaction was found).

SAA upstream of cysteine changed considerably after bariatric surgery (Fig. 1). Methionine concentrations declined $(P<0.001)$, with the lowest concentrations (79\% of presurgery levels) measured 6 months postoperatively. Trends for serum tHcy concentrations differed in the two surgical groups $(P<0 \cdot 001$ for time $\times$ procedure interaction): concentrations increased during weight loss after duodenal switch $(P<0.001)$ but did not change significantly after gastric bypass $(P=0.63)$. Cystathionine dropped steeply from baseline to 6 weeks after surgery (by approximately 36\%) and then remained stable $(P<0 \cdot 001)$. Trends for serum tCys differed according to the surgical procedure $(P=0.02$ for time $\times$ procedure interaction): duodenal switch patients showed a gradual decline in tCys concentrations, reaching $12 \%$ decrease 1 year post-surgery $(P<0 \cdot 001)$, while gastric bypass patients showed no significant change $(P=0 \cdot 22)$. Duodenal switch patients did not have significantly higher tCys than controls 드 1 year after surgery $(P=0 \cdot 11)$.

Biomarkers downstream of cysteine are shown in Fig. 2. No significant changes were found for concentrations of taurine $(P=0.51)$ or $\mathrm{tGSH}(P=0.98)$ during the follow-up. However, glutamate concentrations dropped briskly after surgery $(P<0 \cdot 001)$. Serum GGT activity also decreased $(P<0.01$ in
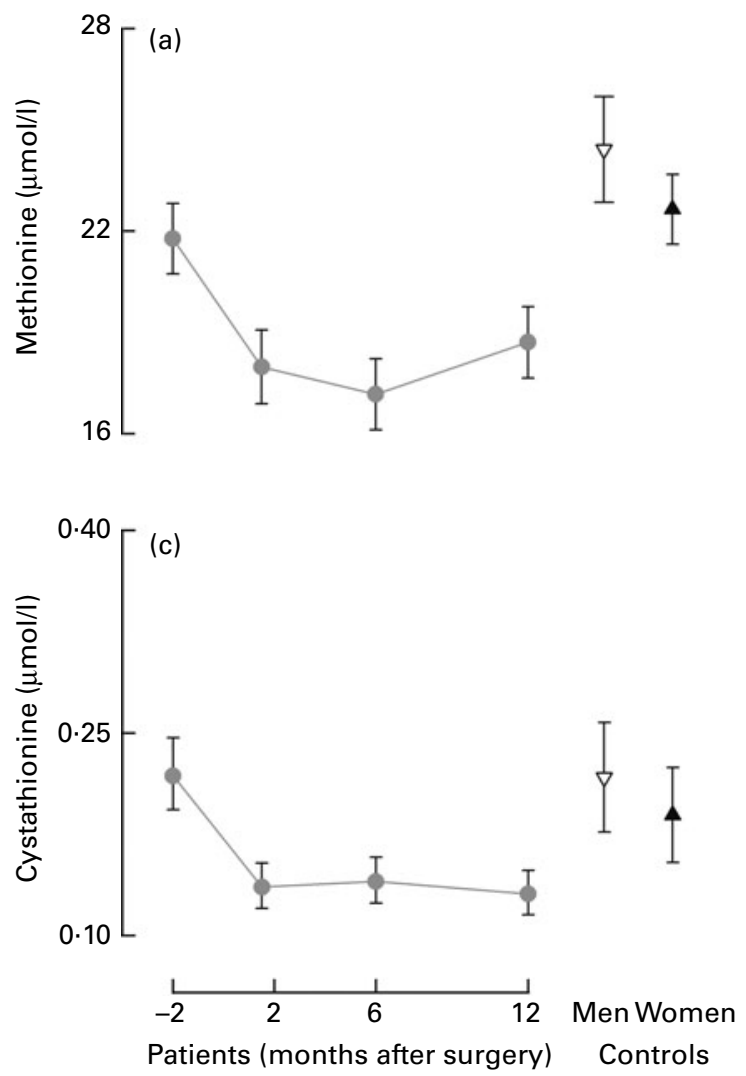

both surgical groups); this trend was more pronounced after gastric bypass than duodenal switch $(P=0.03$ for time $\times$ procedure interaction).

\section{Effect of bariatric surgery on other variables}

For the same patients, we have previously reported 1-year outcomes for the following variables: mean weight loss $(30 \%$ after gastric bypass and $41 \%$ after duodenal switch); serum folate and pyridoxal-5'-phosphate (stable and increased concentrations after both procedures); $\mathrm{Hb}$, total cholesterol and plasma albumin (greater decreases after duodenal switch) ${ }^{(20)}$

Further variables relevant to SAA metabolism are shown in Fig. 3. Serum cobalamin concentrations increased in gastric bypass patients, who received additional vitamin $\mathrm{B}_{12}$ supplementation. Blood FAD concentrations were mostly stable after surgery $(P=0.054)$. Serum creatinine declined $(P<0 \cdot 001)$. Alanine aminotransferase activity increased transiently 6 weeks after surgery, before declining $(P=0.005)$.

\section{Associations of serum total cysteine and related compounds with other variables}

The relationships (Spearman's correlations) of tCys and related compounds with other biomarkers are shown in Table S1
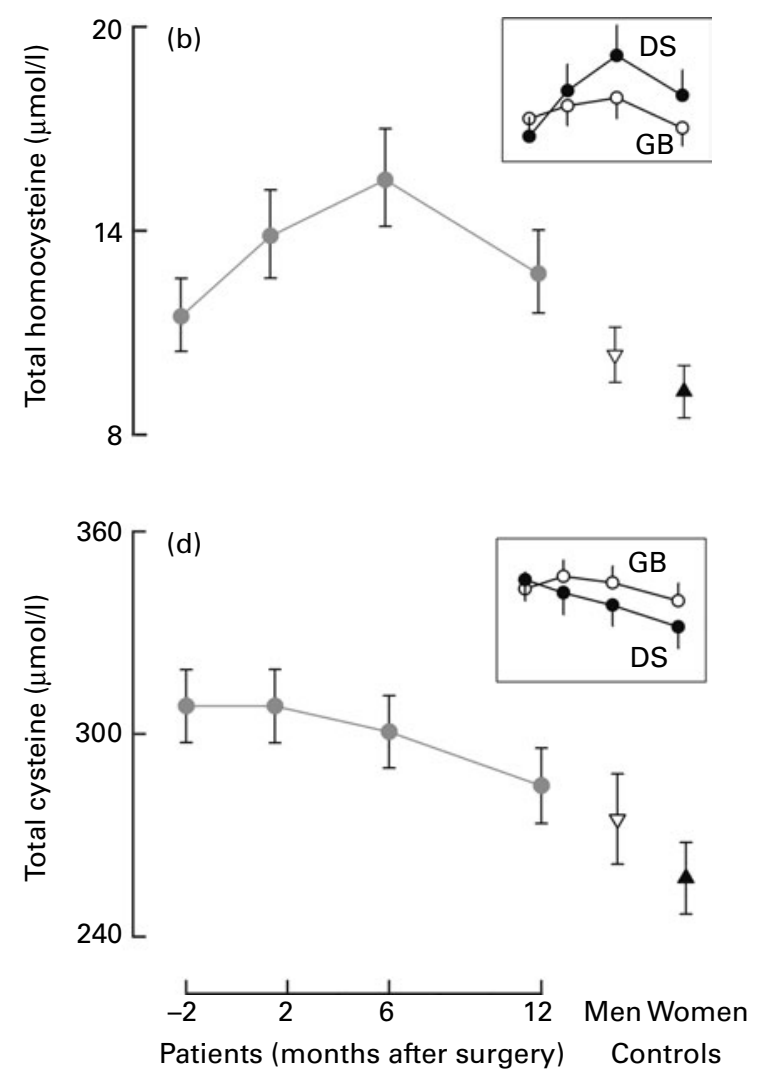

Fig. 1. Biomarkers upstream of cysteine in bariatric surgery patients $(0, n 60)$, healthy men $(\nabla, n 28)$ and healthy women $(\boldsymbol{\Delta}, n 30)$. Linear mixed-effects model: there was a significant trend for (a) methionine ( $\mu \mathrm{mol} / \mathrm{l}), n 30$ (patients, fifteen in each surgical group), (b) total homocysteine ( $\mu \mathrm{mol} / \mathrm{l})$, (c) cystathionine $(\mu \mathrm{mol} / \mathrm{l})$ and (d) total cysteine $(\mu \mathrm{mol} / \mathrm{l})$ after surgery $(P<0.05$, time effect); insets: trend for the two surgical procedures was significantly different $(P<0.05$, time $\times$ procedure interaction). Values are means, with $95 \% \mathrm{Cl}$ represented by vertical bars, adjusted for age, sex and type of surgery in patients and unadjusted in controls. DS (๑), duodenal switch; GB (O), gastric bypass. The precise illustrated patient values are shown in Table S2 of the supplementary material (available online at http://www.journals.cambridge.org/bjn). 

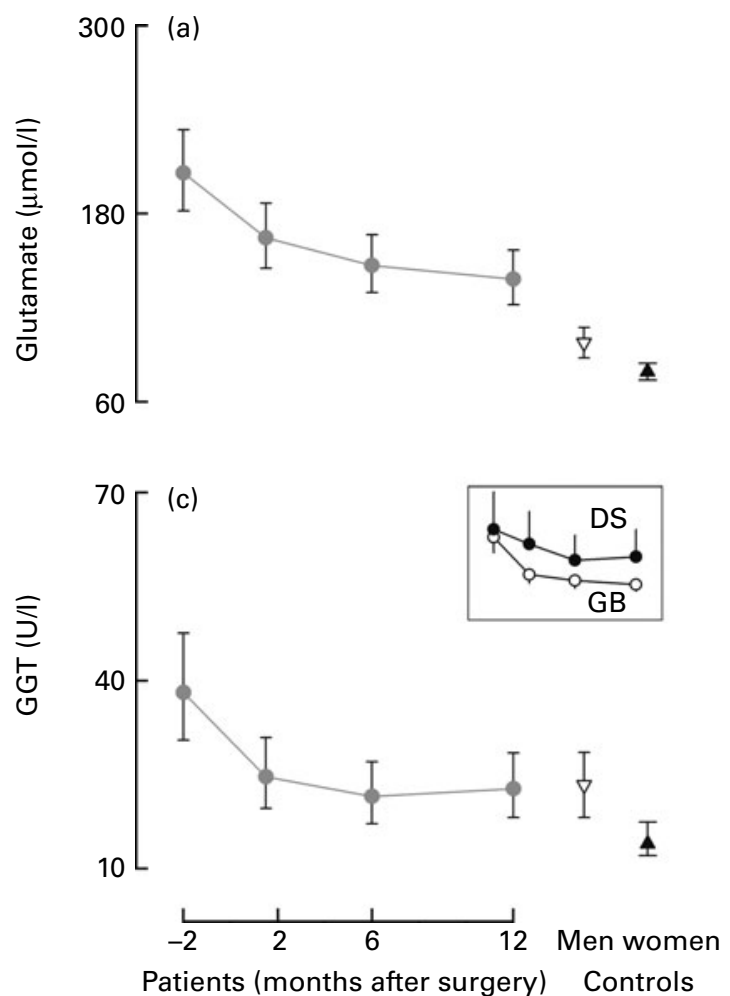
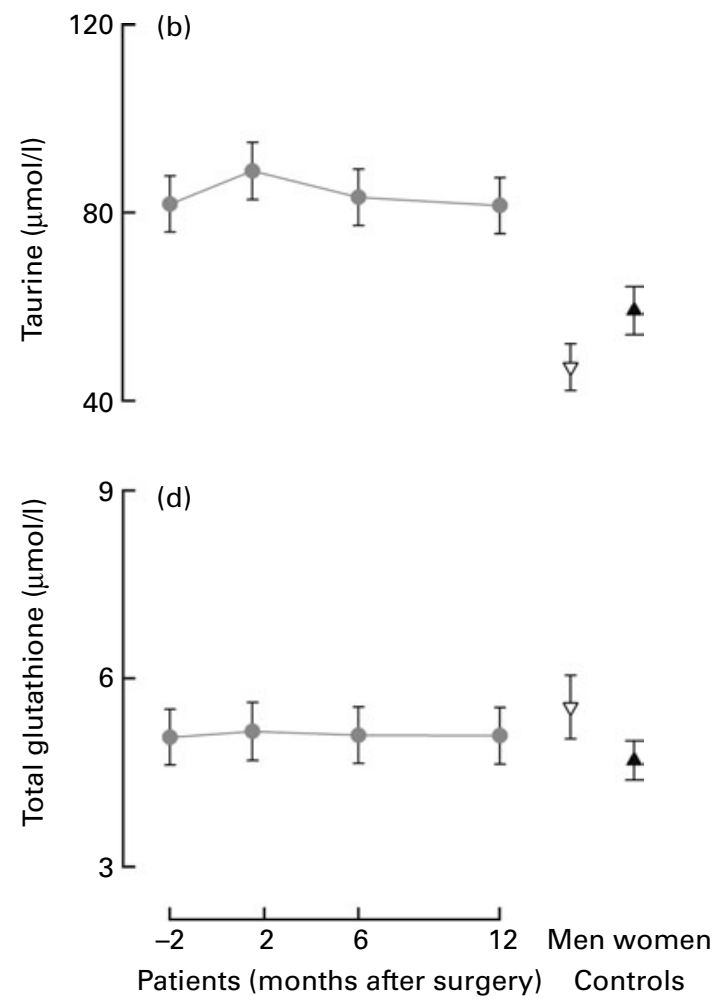

Fig. 2. Biomarkers downstream of cysteine in bariatric surgery patients $(0, n 30)$, healthy men $(\nabla, n 28)$ and healthy women $(\boldsymbol{\Delta}, n 30)$. Linear mixed-effects model: there was a significant trend for (a) glutamate ( $\mu \mathrm{mol} / \mathrm{l})$ and (c) $\gamma$-glutamyltransferase $(\mathrm{GGT})(\mathrm{U} / \mathrm{l})$ after surgery $(P<0.05$, time effect); insets: trend for the two surgical procedures was significantly different $(P<0.05$, time $\times$ procedure interaction). No significant changes observed for $(b)$ taurine $(\mu m o l / l)$ and $(\mathrm{d})$ total glutathione ( $\mu \mathrm{mol} / \mathrm{l})$. DS (๑), duodenal switch; GB (O), gastric bypass. Values are means, with $95 \% \mathrm{Cl}$ represented by vertical bars, adjusted for age, sex and type of surgery in patients and unadjusted in controls. The precise illustrated patient values are shown in Table S2 of the supplementary material (available online at http://www.journals.cambridge.org/bjn).

(supplementary material for this article can be found at http:// www.journals.cambridge.org/bjn). No consistent associations were found with BMI. However, at all patient visits, serum GGT activity correlated positively with glutamate $(\rho=0.79$ to 0.84$)$ and inversely with tGSH ( $\rho=-0.41$ to -0.76$)$ concentrations (Fig. 4). Significant correlations were also noted for creatinine (with tHcy and cystathionine), alanine aminotransferase (with glutamate), folate (with tHcy) and albumin (with tCys).

Mixed-effects models identified the following associations with tCys and related compounds in the patients: age was associated positively with tCys $(P=0.001)$ and inversely with tGSH $(P=0.03)$, while male sex was associated with higher methionine $(P=0.003)$, tHcy $\quad(P=0.002), \quad$ cystathionine $(P=0.02)$ and tCys $(P=0.03)$ concentrations.

\section{Relationship between total cysteine and weight loss}

The relationship between tCys and weight loss was explored in multiple linear regression models, using percentage of weight loss (from baseline to 1 year after surgery) as a dependent variable and surgical procedure (gastric bypass or duodenal switch) as well as baseline BMI, age, sex and tCys as independent variables. In this model $\left(r^{2} 0 \cdot 59\right)$, the only significant predictors of weight loss were surgical procedure and baseline BMI $(P<0 \cdot 001$ for both) (baseline tCys: $P=0 \cdot 43)$.
Replacing baseline tCys with change in tCys (from baseline to 1 year after surgery) did not change the findings $\left(r^{2} 0.59\right.$; change in tCys: $P=0.64$ ).

Total cysteine in bariatric surgery patients and the general population

Finally, we compared the obese patients with a large population-based cohort. Before surgery, the obese patients had a BMI much higher than the 95 th percentile $\left(32 \mathrm{~kg} / \mathrm{m}^{2}\right)$ of the Hordaland Homocysteine Study population, while serum tCys was below the 95th percentile. After surgery, duodenal switch patients had a modest but significant decline in tCys, whereas the gastric bypass patients had no significant change in tCys despite a BMI loss of $16 \cdot 3 \mathrm{~kg} / \mathrm{m}^{2}$ (see Fig. S1 of the supplementary material, available online at http:// www.journals.cambridge.org/bjn).

\section{Discussion}

In the present study, we showed changes in serum tCys and metabolically related amino acids in severely obese patients undergoing surgery-induced weight loss. The patients had modestly elevated tCys concentrations before surgery. At 1 year after surgery, gastric bypass patients showed no significant change in tCys, despite 30\% weight loss. Given the 

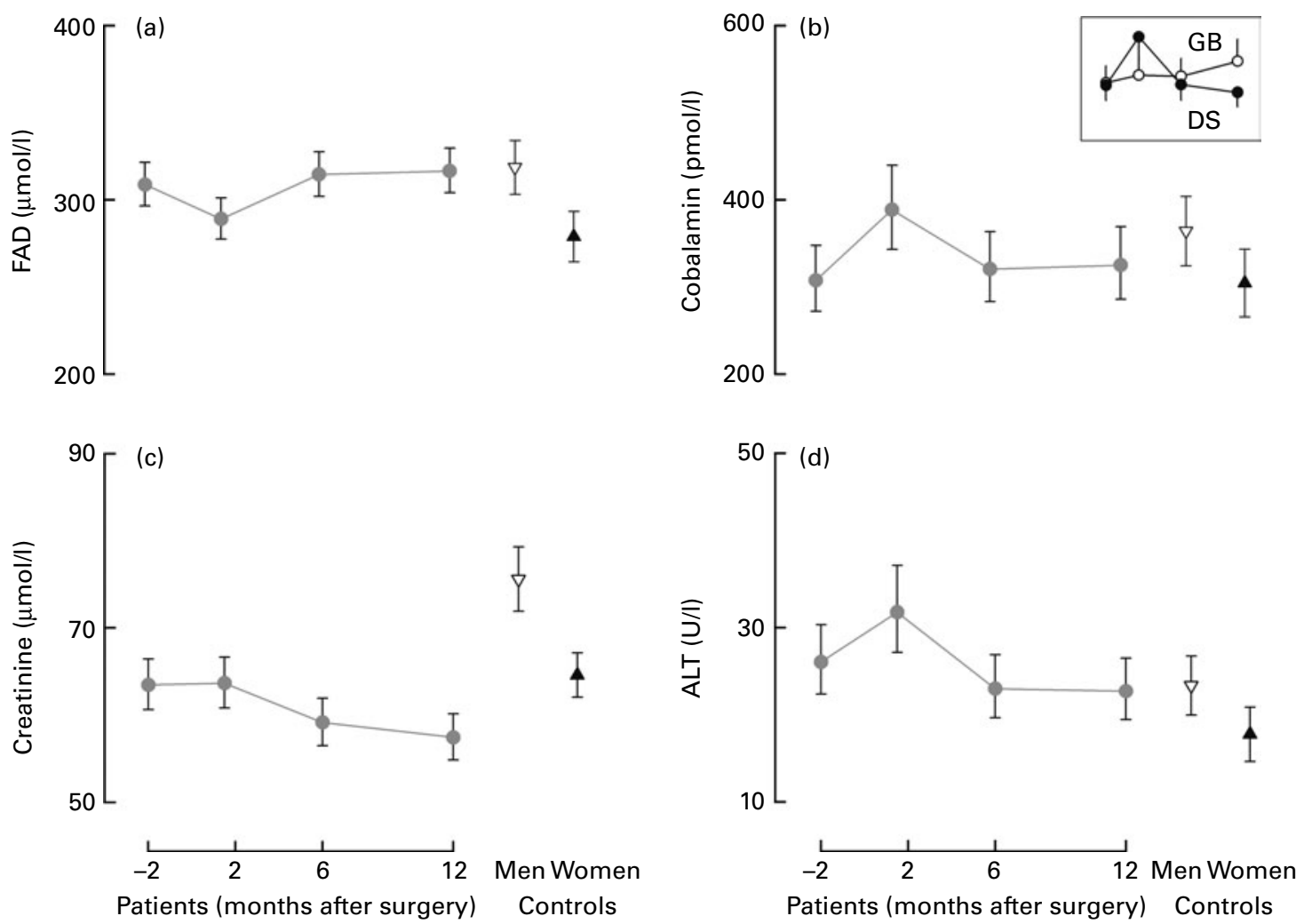

Fig. 3. Biomarkers in bariatric surgery patients $(\bullet, n 60)$, healthy men $(\nabla, n 28)$ and healthy women $(\Lambda, n 30)$. Linear mixed-effects model: there was a significant trend for (b) cobalamin (pmol/l), (c) creatinine $(\mu \mathrm{mol} / \mathrm{l})$ and (d) alanine aminotransferase (ALT, U/l), $n 30$ (patients, fifteen in each surgical group) after surgery $(P<0.05$, time effect); insets: trend for the two surgical procedures was significantly different $(P<0.05$, time $\times$ procedure interaction). No significant changes observed for FAD (nmol/l) (a). Values were means, with $95 \% \mathrm{Cl}$ represented by vertical bars, adjusted for age, sex and type of surgery in patients (cobalamin was additionally adjusted for site) and unadjusted in controls. DS (๑), duodenal switch; GB (O), gastric bypass. The precise illustrated patient values are shown in Table S2 of the supplementary material (available online at http://www.journals.cambridge.org/bjn).

strong association between tCys and fat mass in the general population $^{(6)}$, the present data suggest that fat mass does not cause elevation of tCys, since these very obese patients neither had very high tCys at baseline nor showed a marked drop in tCys after 30\% weight loss. This contrasts with glutamate concentrations, which were markedly elevated before surgery and decreased with both procedures; reflecting the changes in GGT.

It has been known for decades that obese persons may have elevated plasma amino acid levels ${ }^{(25)}$. Given that type of dietary protein influences the risks of having obesity and insulin resistance ${ }^{(26,27)}$, it is conceivable that specific amino acids could contribute to causing these conditions. Recent interest in this field has been fuelled by the finding that certain plasma amino acid profiles, in particular elevated branchedchain amino acid (BCAA) and glutamate levels, may promote insulin resistance in obesity ${ }^{(10,27)}$. Then again, obese rodents with elevated BCAA levels had a reduction in BCAAmetabolising enzymes; and in obese humans, after gastric bypass, a decrease in plasma BCAA was coupled with an increase in BCAA-metabolising enzymes ${ }^{(28)}$. Hence, the elevated plasma BCAA in obesity could be due to disturbances in metabolising enzymes.

The present study targets a different group of amino acids, the SAA. SAA are relevant to clinical obesity not only because of the association of tCys with BMI and fat mass in large cohort studies $^{(4-6)}$, but also because rodent experiments show that restricted intake of methionine, the precursor of all SAA, protects against obesity and insulin resistance ${ }^{(29,30)}$. Furthermore, adding cysteine to a methionine-restricted diet reverses the effect of the diet on rat adiposity and the serum fatty acid profile ${ }^{(31)}$, raising the possibility that cysteine may influence lipid metabolism and weight in humans.

To our knowledge, these are the first data on tCys levels in patients undergoing current bariatric procedures. Early studies found high tCys and glutamic acid levels in obese patients, with varying changes after jejunoileal bypass surgery ${ }^{(32,33)}$. Jejunoileal bypass more often led to metabolic complications (e.g. liver cirrhosis) ${ }^{(34)}$, which may hamper comparison with modern operations. More recent studies have found both increases $^{(15,35)}$ and decreases ${ }^{(36,37)}$ in tHcy in obese patients following bariatric surgery; perhaps related to differences in vitamin supplements, or, as in the present study, the procedure used.

\section{Possible mechanisms for the changes in total cysteine and} related compounds

Methionine and cysteine are normally efficiently digested and absorbed in the intestines, and are removed from the portal blood by the liver for use in the synthesis of proteins and $\mathrm{GSH}$, or for catabolism to taurine ${ }^{(19)}$ (ses Fig. S2 of the 

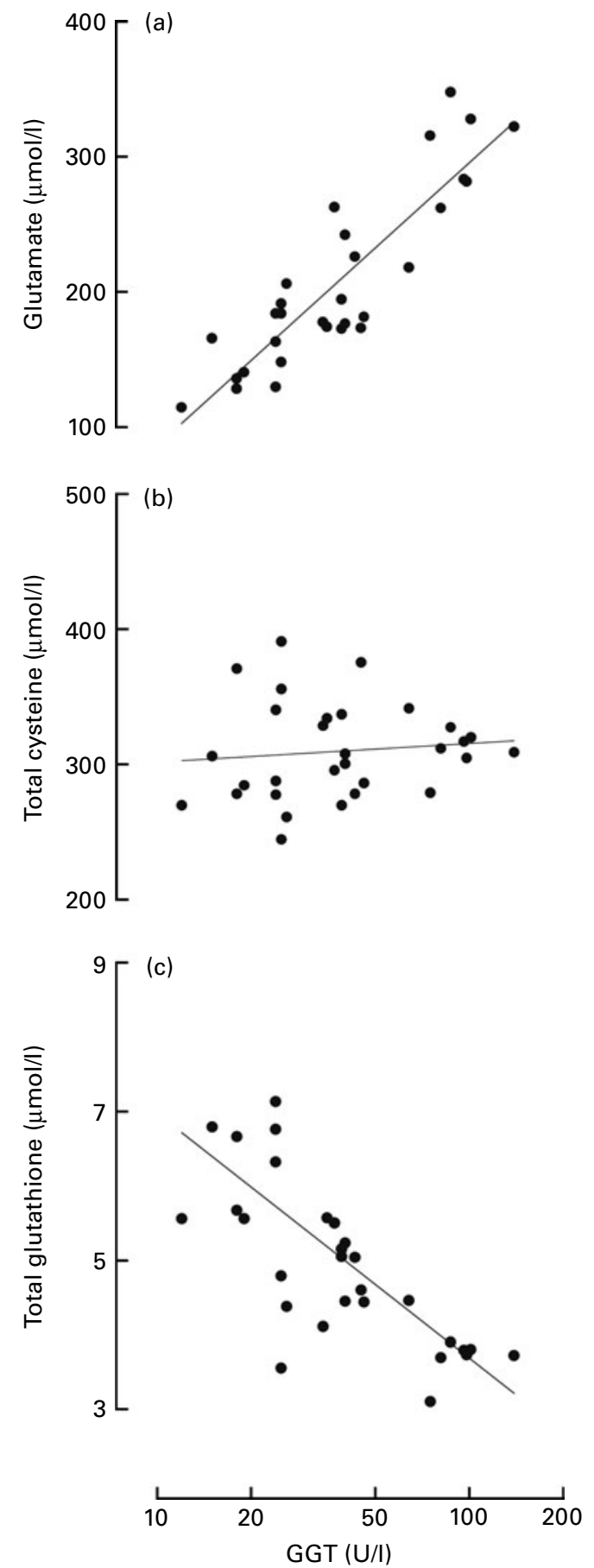

Fig. 4. Relationships between $\gamma$-glutamyltransferase (GGT) and serum concentrations $(\mu \mathrm{mol} / \mathrm{l})$ of (a) glutamate $(r 0.84, P<0.001)$, (b) total cysteine ( $r$ 0.18, $P=0.35$ ), and (c) total glutathione $(r-0.76, P<0.001)$ in obese patients before bariatric surgery $(n 30)$. Spearman's correlation coefficients are shown.

supplementary material, available online at http://www. journals.cambridge.org/bjn). While gastric bypass patients presumably lose weight mainly by lowering energy intake, biliopancreatic diversion with duodenal switch causes more protein and fat malabsorption ${ }^{(38,39)}$ and more often leads to nutrient deficiencies ${ }^{(20)}$. Protein depletion and methionine restriction lead to impaired cystathionine $\beta$-synthase enzyme activity: this limits trans-sulphuration of homocysteine to cystathionine to preserve methionine ${ }^{(40,41)}$. Sparse protein availability in duodenal switch patients could thus help explain their higher tHcy concentrations and, secondary to diminished flux through the trans-sulphuration pathway, their lower tCys concentrations. Elevated tHcy could potentially in itself also contribute to decreasing serum tCys by displacing protein-bound cysteine from albumin ${ }^{(42)}$. Finally, given that the gastrointestinal tract is an active site for SAA metabolism $^{(43)}$, it is possible that differences in tCys after gastric bypass and duodenal switch could in part relate to varying intestinal SAA metabolism following these procedures.

Changes in dietary intakes and food preferences ${ }^{(44)}$ and use of vitamin supplements could have contributed to changes in SAA concentrations after surgery. It has been suggested that higher serum cobalamin and folate levels are needed to maintain tHcy during weight loss ${ }^{(15)}$. Cobalamin is a cofactor in homocysteine remethylation to methionine ${ }^{(1)}$. Duodenal switch patients did not receive parenteral cobalamin supplementation, so a relative cobalamin deficiency may have contributed to the elevation of their tHcy concentrations. Both surgical groups showed increases in serum vitamin $\mathrm{B}_{6}$ postoperatively, which probably explains the abrupt $36 \%$ reduction in cystathionine concentrations after surgery ${ }^{(45)}$ : vitamin $\mathrm{B}_{6}$ supplementation increases the conversion of cystathionine to cysteine by the cystathionine $\gamma$-lyase enzyme, which is very sensitive to pyridoxal- $5^{\prime}$-phosphate depletion $^{(46)}$.

Taurine can be obtained from the diet, or via conversion of cysteine by cysteine dioxygenase ${ }^{(47)}$. Since cysteine dioxygenase is up-regulated in the liver and adipose tissue in response to high cysteine availability ${ }^{(19)}$, the higher taurine concentrations in obese patients compared with controls could be secondary to increased conversion of cysteine to taurine. Glutamate was much higher in obese patients than controls, as shown by others ${ }^{(9,10)}$, and decreased after surgery. Elevated GGT levels in obesity, and their decrease during weight loss, also correspond with previous research ${ }^{(14)}$. Perhaps surprisingly, despite losing more weight, duodenal switch patients had higher GGT levels than gastric bypass patients during the follow-up. This could reflect either higher alcohol intake, or less regression of non-alcoholic fatty liver disease ${ }^{(48)}$ in duodenal switch patients relative to gastric bypass patients post-surgery. Strong correlations were found for GGT with tGSH (inverse) and glutamate (positive) before and after surgery. Despite a decline in GGT activity, however, patients had stable tGSH concentrations. Other researchers reported increased erythrocyte GSH after gastric banding ${ }^{(49)}$. The varying results could relate to our assessment of tGSH in the serum; and not erythrocytes, where GSH is more abundant. Our finding of a strong correlation $(r$ 0.8) between serum GGT activity and glutamate concentrations may reflect the role of GGT in hydrolysing GSH to ultimately yield glutamate (and cysteine), and could suggest that GGT influences plasma glutamate. Increased glutamate levels in obesity may thus be secondary to disturbances in metabolic enzymes, similar to $\mathrm{BCAA}^{(28)}$. 
A longitudinal study of predictors of change in tCys in a population-based cohort showed that a decrease in BMI of $1 \mathrm{~kg} / \mathrm{m}^{2}$ or more was associated with a corresponding decrease in tCys $^{(7)}$. In the present trial, despite $30 \%$ weight loss, there was no significant change in tCys after gastric bypass surgery. This suggests that the epidemiological association between changes in tCys and changes in $\mathrm{BMI}^{(7)}$ is not explained by an effect of body weight on plasma tCys levels. The $12 \%$ decrease in tCys seen in the present study after duodenal switch surgery may relate to additional malabsorption with this procedure ${ }^{(38,39)}$. Consistent with this, duodenal switch patients had lower serum albumin than gastric bypass patients ${ }^{(20)}$, and serum albumin correlated positively with tCys in the combined patient group.

The strengths of the present study include a broad biochemical characterisation of patients throughout major weight loss. Unlike previous reports on other amino $\operatorname{acids}^{(28)}$, we did not investigate changes in metabolic enzymes, apart from plasma GGT. However, the serial examinations enabled us to distinguish between amino acid changes that occurred rapidly (e.g. decrease in cystathionine) or more gradually (e.g. decrease in glutamate) during weight loss. The randomised trial design allowed us to compare the effects of two different surgical techniques. We did not assess dietary SAA content, but monitored the intake of dietary supplements ${ }^{(20)}$, and use of fasting blood samples minimised bias from acute variations in SAA intake ${ }^{(50)}$.

In summary, the stability of tCys during weight loss contrasts with the marked drops in serum methionine, cystathionine and glutamate. This may illustrate that elevated plasma amino acid levels associated with obesity fall into at least two categories: those that are an adjunct to obesity and those that may contribute towards the development of obesity. This can potentially have implications for the modulation of dietary protein content in future strategies to prevent obesity.

\section{Acknowledgements}

The authors' responsibilities were as follows: E. T. A. (guarantor), A. K. E. and H. R. organised the amino acid studies; E. T. A., T. T. S., T. M. and T. O. organised the clinical trial; E. T. A. and K. I. B. organised examination of the healthy controls; M. V.-G. performed the amino acid assays; E. T. A., A. K. E., L. M. D. and H. R. cross-checked and analysed the data; E. T. A. and L. M. D. performed the statistical analyses; E. T. A. and A. K. E. wrote the manuscript; L. M. D., T. T. S., T. M., M. V.-G., T. O., T. B., K. I. B. and H. R. revised the manuscript; E. T. A., T. B. and H. R. obtained funding; H. R. supervised the amino acid studies. The authors acknowledge Carl Fredrik Schou, Jon Kristinsson and Hans Lönroth (bariatric surgery); and My Engström and Kari Julien (biobanking). The present study was supported by a research fellowship grant from the South Eastern Norway Regional Health Authority and research grants from the South Eastern Norway Regional Health Authority (AUS-20-2006) and Aker University Hospital (08/835 and 09/40) (all to E. T. A.), and the Norwegian Research Council (197195/HR). The sponsors had no role in designing the study or in collecting, analysing or interpreting the data. The authors declare that they have no conflict of interest.

\section{References}

1. Brosnan JT \& Brosnan ME (2006) The sulfur-containing amino acids: an overview. J Nutr 136, 1636S-1640S.

2. Byington MH, Howe JM \& Clark HE (1972) Effect of different levels and proportions of methionine, cystine, choline, and inorganic sulfur on growth and body composition of young rats. J Nutr 102, 219-227.

3. Sarwar Gilani G, Nimal Ratnayake WM, Peace RW, et al (2006) Effects of supplemental cystine or methionine on growth and lifespan of stroke-prone spontaneously hypertensive rats. Br J Nutr 95, 443-447.

4. El-Khairy L, Ueland PM, Nygard O, et al. (1999) Lifestyle and cardiovascular disease risk factors as determinants of total cysteine in plasma: the Hordaland Homocysteine Study. Am J Clin Nutr 70, 1016-1024.

5. Elshorbagy AK, Refsum H, Smith AD, et al. (2009) The association of plasma cysteine and gamma-glutamyltransferase with BMI and obesity. Obesity (Silver Spring) 17, $1435-1440$.

6. Elshorbagy AK, Nurk E, Gjesdal CG, et al. (2008) Homocysteine, cysteine, and body composition in the Hordaland Homocysteine Study: does cysteine link amino acid and lipid metabolism? Am J Clin Nutr 88, 738-746.

7. El-Khairy L, Vollset SE, Refsum H, et al. (2003) Predictors of change in plasma total cysteine: longitudinal findings from the Hordaland Homocysteine Study. Clin Chem $\mathbf{4 9}$, $113-120$.

8. Kennedy BP, Rao F, Botiglieri T, et al. (2005) Contributions of the sympathetic nervous system, glutathione, body mass and gender to blood pressure increase with normal aging: influence of heredity. J Hum Hypertens 19, 951-969.

9. Proenza AM (1998) Blood amino acid compartmentation in men and women with different degrees of obesity. J Nutr Biochem 9, 697-704.

10. Newgard CB, An J, Bain JR, et al. (2009) A branched-chain amino acid-related metabolic signature that differentiates obese and lean humans and contributes to insulin resistance. Cell Metab 9, 311-326.

11. Lu SC (2009) Regulation of glutathione synthesis. Mol Aspects Med 30, 42-59.

12. Whitfield JB (2001) Gamma glutamyl transferase. Crit Rev Clin Lab Sci 38, 263-355.

13. Lee DH, Silventoinen K, Jacobs DR, et al. (2004) Gamma-glutamyltransferase, obesity, and the risk of type 2 diabetes: observational cohort study among 20,158 middle-aged men and women. J Clin Endocrinol Metab 89, 5410-5414.

14. Dixon JB, Bhathal PS \& O'Brien PE (2006) Weight loss and non-alcoholic fatty liver disease: falls in gamma-glutamyl transferase concentrations are associated with histologic improvement. Obes Surg 16, 1278-1286.

15. Dixon JB, Dixon ME \& O'Brien PE (2001) Elevated homocysteine levels with weight loss after Lap-Band surgery: higher folate and vitamin $\mathrm{B}_{12}$ levels required to maintain homocysteine level. Int J Obes Relat Metab Disord 25, 219-227.

16. Elshorbagy AK, Valdivia-Garcia M, Refsum H, et al. (2010) Sulfur amino acids in methionine-restricted rats: hyperhomocysteinemia. Nutrition 26, 1201-1204.

17. Pollack PF, Rivera A Jr, Rassin DK, et al. (1996) Cysteine supplementation increases glutathione, but not polyamine, concentrations of the small intestine and colon of 
parenterally fed newborn rabbits. I Pediatr Gastroenterol Nutr 22, 364-372.

18. Tozer RG, Tai P, Falconer W, et al. (2008) Cysteine-rich protein reverses weight loss in lung cancer patients receiving chemotherapy or radiotherapy. Antioxid Redox Signal 10, 395-402.

19. Stipanuk MH, Ueki I, Dominy JE, et al. (2009) Cysteine dioxygenase: a robust system for regulation of cellular cysteine levels. Amino Acids 37, 55-63.

20. Aasheim ET, Bjorkman S, Sovik TT, et al. (2009) Vitamin status after bariatric surgery: a randomized study of gastric bypass and duodenal switch. Am J Clin Nutr 90, 15-22.

21. Aasheim ET, Hofso D, Hjelmesaeth J, et al. (2008) Vitamin status in morbidly obese patients: a cross-sectional study. Am J Clin Nutr 87, 362-369.

22. Antoniades C, Shirodaria C, Leeson P, et al. (2009) MTHFR $677 \mathrm{C}>\mathrm{T}$ polymorphism reveals functional importance for 5-methyltetrahydrofolate, not homocysteine, in regulation of vascular redox state and endothelial function in human atherosclerosis. Circulation 119, 2507-2515.

23. Refsum H, Johnston C, Guttormsen AB, et al. (2006) Holotranscobalamin and total transcobalamin in human plasma: determination, determinants, and reference values in healthy adults. Clin Chem 52, 129-137.

24. R Foundation for Statistical Computing (2008) R: A Language and Environment for Statistical Computing. Vienna: R Foundation for Statistical Computing.

25. Felig P, Marliss E \& Cahill GF Jr (1969) Plasma amino acid levels and insulin secretion in obesity. $N$ Engl J Med 281, 811-816.

26. Rosell M, Appleby P, Spencer E, et al. (2006) Weight gain over 5 years in 21,966 meat-eating, fish-eating, vegetarian, and vegan men and women in EPIC-Oxford. Int $J$ Obes (Lond) 30, 1389-1396.

27. Tremblay F, Lavigne C, Jacques H, et al. (2007) Role of dietary proteins and amino acids in the pathogenesis of insulin resistance. Annu Rev Nutr 27, 293-310.

28. She P, Van Horn C, Reid T, et al. (2007) Obesity-related elevations in plasma leucine are associated with alterations in enzymes involved in branched-chain amino acid metabolism. Am J Physiol Endocrinol Metab 293, E1552-E1563.

29. Rizki G, Arnaboldi L, Gabrielli B, et al. (2006) Mice fed a lipogenic methionine-choline-deficient diet develop hypermetabolism coincident with hepatic suppression of SCD-1. J Lipid Res 47, 2280-2290.

30. Malloy VL, Krajcik RA, Bailey SJ, et al. (2006) Methionine restriction decreases visceral fat mass and preserves insulin action in aging male Fischer 344 rats independent of energy restriction. Aging Cell 5, 305-314.

31. Elshorbagy AK, Valdivia-Garcia M, Mattocks DA, et al. (2011) Cysteine supplementation reverses methionine restriction effects on rat adiposity: significance of stearoyl-coenzyme A desaturase. J Lipid Res 52, 104-112.

32. Backman L, Hallberg D \& Kallner A (1975) Amino acid pattern in plasma before and after jejuno-ileal shunt operation for obesity. Scand J Gastroenterol 10, 811-816.

33. Kihlberg R, Bark S \& Hallberg D (1982) An oral amino acid loading test before and after intestinal bypass operation for morbid obesity. Acta Chir Scand 148, 73-86.

34. Hocking MP, Duerson MC, O'Leary JP, et al. (1983) Jejunoileal bypass for morbid obesity. Late follow-up in 100 cases. N Engl J Med 308, 995-999.
35. Borson-Chazot F, Harthe C, Teboul F, et al. (1999) Occurrence of hyperhomocysteinemia 1 year after gastroplasty for severe obesity. J Clin Endocrinol Metab 84, 541-545.

36. Sledzinski T, Goyke E, Smolenski RT, et al. (2009) Decrease in serum protein carbonyl groups concentration and maintained hyperhomocysteinemia in patients undergoing bariatric surgery. Obes Surg 19, 321-326.

37. Williams DB, Hagedorn JC, Lawson EH, et al. (2007) Gastric bypass reduces biochemical cardiac risk factors. Surg Obes Relat Dis 3, 8-13.

38. Puzziferri N, Blankenship J \& Wolfe BM (2006) Surgical treatment of obesity. Endocrine 29, 11-19.

39. Scopinaro N, Marinari G, Camerini G, et al. (2005) Biliopancreatic diversion for obesity: state of the art. Surg Obes Relat Dis 1, 317-328.

40. Ingenbleek Y, Hardillier E \& Jung L (2002) Subclinical protein malnutrition is a determinant of hyperhomocysteinemia. Nutrition 18, 40-46.

41. Tang B, Mustafa A, Gupta S, et al. (2009) Methioninedeficient diet induces post-transcriptional downregulation of cystathionine beta-synthase. Nutrition 26, 1170-1175.

42. Mansoor MA, Svardal AM, Schneede J, et al. (1992) Dynamic relation between reduced, oxidized, and protein-bound homocysteine and other thiol components in plasma during methionine loading in healthy men. Clin Chem 38, 1316-1321.

43. Bauchart-Thevret C, Stoll B \& Burrin DG (2009) Intestinal metabolism of sulfur amino acids. Nutr Res Rev 22, 175-187.

44. Olbers T, Bjorkman S, Lindroos A, et al. (2006) Body composition, dietary intake, and energy expenditure after laparoscopic Roux-en-Y gastric bypass and laparoscopic vertical banded gastroplasty: a randomized clinical trial. Ann Surg 244, 715-722.

45. Bleie O, Refsum H, Ueland PM, et al. (2004) Changes in basal and postmethionine load concentrations of total homocysteine and cystathionine after B vitamin intervention. $\mathrm{Am} \mathrm{J}$ Clin Nutr 80, 641-648.

46. Martinez M, Cuskelly GJ, Williamson J, et al. (2000) Vitamin B-6 deficiency in rats reduces hepatic serine hydroxymethyltransferase and cystathionine beta-synthase activities and rates of in vivo protein turnover, homocysteine remethylation and transsulfuration. $J$ Nutr 130, 1115-1123.

47. Ueki I \& Stipanuk MH (2009) 3T3-L1 adipocytes and rat adipose tissue have a high capacity for taurine synthesis by the cysteine dioxygenase/cysteinesulfinate decarboxylase and cysteamine dioxygenase pathways. J Nutr 139, 207-214.

48. Mathurin P, Hollebecque A, Arnalsteen L, et al. (2009) Prospective study of the long-term effects of bariatric surgery on liver injury in patients without advanced disease. Gastroenterology 137, 532-540.

49. Uzun H, Konukoglu D, Gelisgen R, et al. (2007) Plasma protein carbonyl and thiol stress before and after laparoscopic gastric banding in morbidly obese patients. Obes Surg $\mathbf{1 7}$, $1367-1373$.

50. Guttormsen AB, Solheim E \& Refsum H (2004) Variation in plasma cystathionine and its relation to changes in plasma concentrations of homocysteine and methionine in healthy subjects during a 24-h observation period. Am J Clin Nutr 79, 76-79. 